\title{
Passive Viscous Haptic Textures
}

\author{
Gianni Campion* Andrew H. C. Gosline $\quad$ Vincent Hayward* \\ Haptics Laboratory, McGill University \\ Montréal, Québec, Canada
}

\begin{abstract}
We describe the use of eddy current brakes for the synthesis haptic textures. Textural effects are achieved through rapid variations of the viscous damping coefficient that these brakes create when activated. We demonstrate that eddy current brakes can be actuated reliably at frequencies typical of haptic texture rendering. Performance is evaluated by measuring the movement of the manipulandum with an accelerometer while modulating the viscous force at high frequency. Key advantages of this technique include guaranteed passivity of the haptic synthesis, accurate results from the linear dynamics of these brakes, and elimination of the need to estimate or observe velocity.
\end{abstract}

Keywords: Haptic rendering, haptic devices, haptic textures

Index Terms: H.5.1 [Information Interfaces and Presentation]: Multimedia Information Systems-Artificial, augmented, and virtual realities H.5.2 [Information Interfaces and Presentation]: User Interfaces-Haptic I/O

\section{INTRODUCTION}

Virtual reality applications are becoming ubiquitous in both academic and industrial settings. The quality of this virtual experience is improved by allowing a multisensorial experience of the synthetic world; real time, high-fidelity haptic rendering is a fundamental element of such immersive systems. Haptic texture rendering has the potential for drammatically improving the quality of the virtual world, however, the problem of rendering textures on force feedback devices presents some challenges [2].

Recent advances in passive actuation design made it possible to produce fast varying viscosity fields [4]. Their application to virtual textures is explored here. Such textures can be generated by applying a resistive force proportional to the velocity of the interface, usually of the form $f=-b(x) v$. The perceptual qualities of viscosity-based textures have been investigated by Wiesenberger $e t$ al. and Lederman et al. $[6,5]$. Their findings indicate that the viscosity coefficient greatly influences the perception of roughness and that viscous textures are more detectable than spring-based textures. It should be noted that these prior perceptual evaluations were carried out with force feedback devices actuated by motors.

The rendering of viscous fields with active actuators requires an estimate, $\hat{v}$, of the velocity at which the manipulandum is moving. Such task is usually accomplished by differentiating the position signal from encoders. A force proportional to this velocity, $-b(\hat{x}) \hat{v}$, is then presented to the user. It is well known that a velocity signal estimated from a position signal can be either noisy and discontinuous or smooth but delayed. In both cases, rendering with a velocity signal can produce undesirable artifacts. With eddy current brakes

\footnotetext{
*e-mail: champ@ cim.mcgill.ca

†e-mail:andrewg@cim.mcgill.ca

‡e-mail:hayward@cim.mcgill.ca
}

(ECB), which by physics provide a braking force proportional to velocity, the issues related to velocity computation are eliminated.

In this paper we show that the eddy current dampers can provide adequate frequency response. We also verify experimentally that the varying viscosity field is converted into acceleration variations on the manipuladum. Finally we demonstrate that textures can be rendered when exploring a passive, varying viscous friction field.

\section{EXPERIMENTAL SETUP}

The experiments are performed on a 1-DOF haptic interface shown in Fig. 1. This simplified interface comprises a DC coreless motor (RE25, Maxon ag), a 65 kCPR incremental encode (Gurley Precision Instruments), a solid state accelerometer (Analog Devices ADXL311JE), a PWM current amplifier 25A20 (Advanced Motion Controls) and a custom made eddy current brake. Control of the interface and data aquisition are performed by a $2.4 \mathrm{GHz}$ PC with a Q8 DAC (Q8, Quanser Inc.) running the Linux kernel 2.6 and the Xenomai Real-Time Framework. The sampling rate is $5 \mathrm{kHz}$.

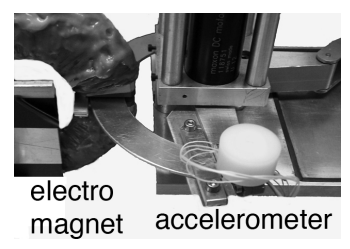

Figure 1: Hardware. One of the two proximal arms $(63 \mathrm{~mm})$ of the Pantograph [3] was disconnected to be used as a manipuladum. The eddy current brake is an improved version of the design in [4]. The accelerometer is attached at $51 \mathrm{~mm}$ away from the axis of rotation.

\section{RESULtS}

\subsection{Response of Current Excitation}

The frequency response of current excitation was measured with a system analyzer (SigLab model 20-22, DSP Technology Inc.). Figure 2 shows the response of the current in the coil as a function of the command signal to the current amplifiers. The response show a progressive roll-off from $\mathrm{DC}$ to $1 \mathrm{kHz}$ and less than $3 \mathrm{~dB}$ of attenuation from $10 \mathrm{~Hz}$ to $100 \mathrm{~Hz}$. For viscous texture rendering, the response under $10 \mathrm{~Hz}$ is not significant since low frequency viscosity modulation does not give a "textural" effect.

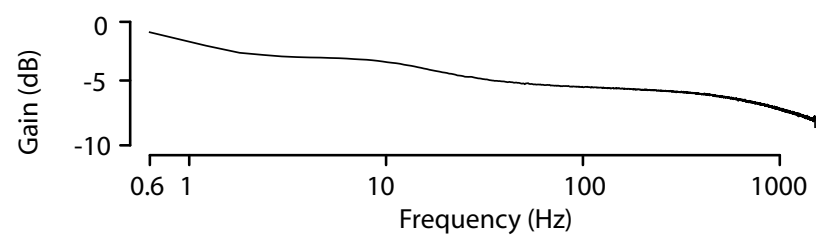

Figure 2: Current drive response on inductive load. 


\subsection{Response from Current Excitation to Acceleration}

The device creates a resistive torque $\tau=-b(t) \omega$ where $\omega$ is the angular velocity of the arm around the rotation joint. To very good approximation, the viscous damping coefficient is proportional to current: $b(t)=B i(t)[4]$, where $B$ is a characteristic of the damper. First, the current in the coils, $i(t)$, is pulsed according to

$$
i(t)=I[1+\sin (2 \pi v t)] .
$$

Figure 3, obtained with $I=2 \mathrm{~A}$ and $v=50,100 \mathrm{~Hz}$, shows the spectrum of the acceleration signal recorded when moving the handle manually. The large, low frequency peak corresponds to the low frequency almost-periodic hand movements (between 1 and $3 \mathrm{~Hz}$ ). In the high frequencies, instead of having one single peak corresponding to the braking force pulsing ( 50 and $100 \mathrm{~Hz}$ respectively), two sidebands appear around the carrier frequency. These sidebands correspond to the modulation of a high frequency carrier (the pulsing) by a low frequency signal (the movement) [1].

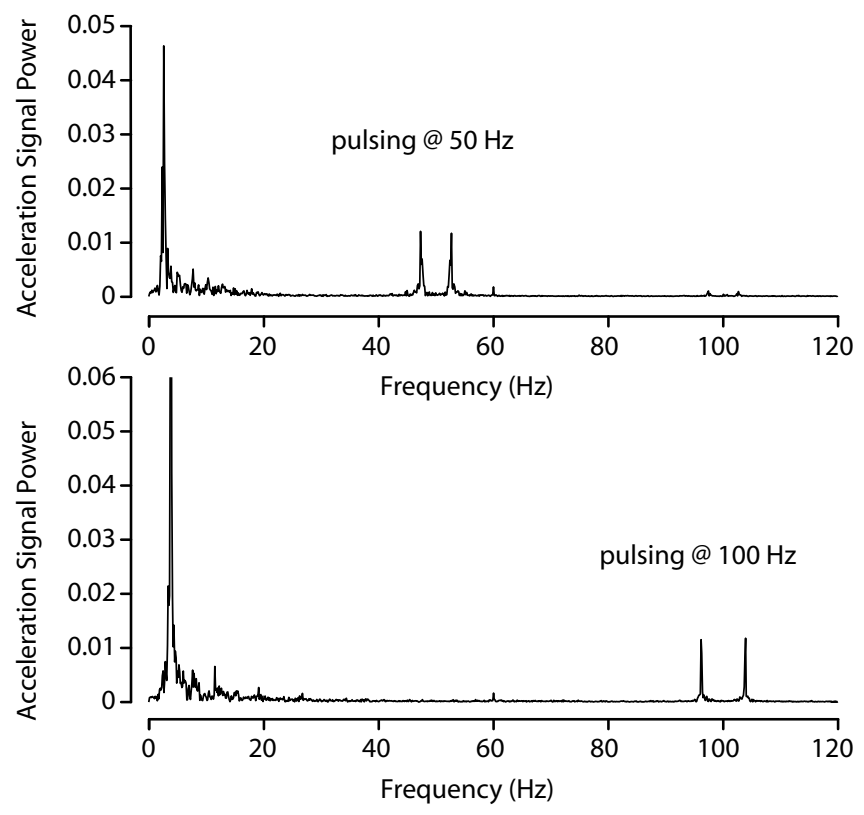

Figure 3: Spectrum (FFT) of acceleration signal with pulsed braking. There is a small $60 \mathrm{~Hz}$ noise component.

A portion of the time-domain acceleration signal is in Figure 4. When acceleration peaks, velocity is zero and the texture vanishes.

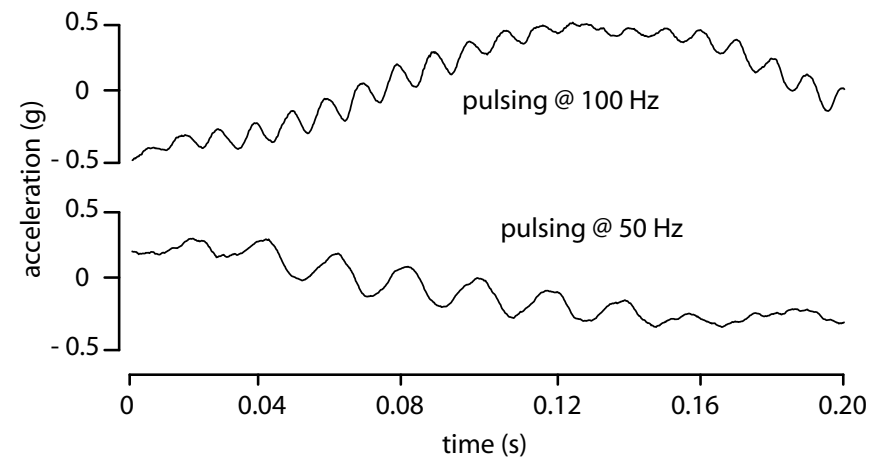

Figure 4: Portion of acceleration signal at the tip in the time domain.

\subsection{Spatially Coded Texture}

An instance of a spatially varying viscous coefficient can be written

$$
i=I(1+\sin [50 \pi \theta])
$$

where $I=2$ A. Figure 5 shows the resulting acceleration signal when scanning. A five samples running average is applied to reduce sensor noise. When acceleration goes through zero, velocity is maximum. As may be expected, these velocity variations change the period of the texture.

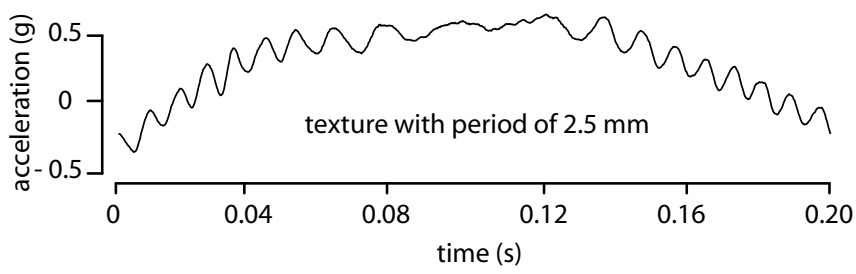

Figure 5: Acceleration at the tip recording for a viscous texture.

\section{Feasibility of ECB Generated Textures and Fu- TURE CONSIDERATIONS}

Viscosity fields have been used previously to convey textural information. Computer-controlled eddy-current brakes are now shown to be capable of generating such fields at temporal frequencies higher than least $100 \mathrm{~Hz}$. The resulting manipulandum movements are by physics, continuous, passive, predictable, and yet can contain high frequency components. They do not suffer from the injurious effects of quantization and sampling which often plague haptic simulation that rely on active force feedback to generate such fields. Future work will include the synthesis of a richer set of textures by viscosity modulation. Another promising avenue is in the combination of active force feedback with viscous braking.

\section{ACKNOWLEDGEMENTS}

This work was funded by NSERC, the Natural Sciences and Engineering Council of Canada, and Immersion Corporation.

\section{REFERENCES}

[1] H. S. Black. Modulation Theory. Van Nostrand, 1953.

[2] G. Campion and V. Hayward. Fundamental limits in the rendering of virtual haptic textures. In Proceedings of the First Joint Eurohaptics Conference and Symposium on Haptic Interfaces for Virtual Environment and Teleoperator Systems, World Haptics 2005, pages 263-270, 2005.

[3] G. Campion, Q. Wang, and V. Hayward. The Pantograph Mk-II: A haptic instrument. In Proceedings of the IEEE/RSJ International Conference on Intelligent Robots and Systems, IROS'05, pages 723-728, 2005.

[4] A. H. Gosline, G. Campion, and V. Hayward. On the use of eddy current brakes as tunable, fast turn-on viscous dampers for haptic rendering. In Proceedings of Eurohaptics, pages 229-234, 2006.

[5] S. J. Lederman, R. L. Klatzky, C. Tong, and C. Hamilton. The perceived roughness of resistive virtual textures: II. effects of varying viscosity with a force-feedback device. ACM Trans. Appl. Percept., 3(1):15-30, 2006.

[6] J. M. Weisenberger, M. J. Kreier, and M. A. Rinker. Judging the orientation of sinusoidal and square-wave virtual gratings presented via 2-DOF and 3-DOF haptic interfaces. Haptics-e, 1(4), 2000. 